\title{
SINERGISMO IN VITRO DA ASSOCIAÇÃO ENTRE PRALIDOXIMA E SULFONAMIDAS NA REATIVAÇÃO DA ACETILCOLINESTERASE HUMANA RECOMBINANTE INIBIDA POR PARAOXON
}

\author{
Rômulo Santiago de Lima GARCIA* \\ Instituto de Defesa Química, Biológica, Radiológica e Nuclear - Seção de Defesa Biológica. Rio de Janeiro, Brasil. \\ *Autor para correspondência: romulosantiagodelimagarcia@gmail.com
}

DOI: http://dx.doi.org/10.18571/acbm.131

\section{RESUMO}

Foi realizada a avaliação da capacidade de reativação da acetilcolinesterase humana recombinante inibida previamente com solução de Paraoxon $2 \mu \mathrm{M}$ diante de pralidoxima em solução a $100 \mu \mathrm{M}$ em associação com onze derivados de sulfonamida em solução a $100 \mu \mathrm{M}$ em dimetilsulfóxido, a saber, $\mathrm{N}$-(2-clorofenil)benzenossulfonamida，N-(4-fluorofenil)benzenossulfonamida， N-(4-fluorofenil)-4nitrobenzenossulfonamida, $\quad$ 4-cloro-N-(4-fluorofenil)benzenossulfonamida, $\quad \mathrm{N}$-(3clorofenil)benzenossulfonamida, $\quad \mathrm{N}$-(3-cloro-2-metilfenil)benzenossulfonamida, 4-cloro-Nfenilbenzenossulfonamida, $\quad \mathrm{N}$-(2-clorofenil)-4-metilbenzenossulfonamida, $\mathrm{N}$-(3-clorofenil)-4metilbenzenossulfonamida, $\quad \mathrm{N}$-(2-hidroxietil)-4-metil-N-fenilbenzenossulfonamida e $\mathrm{N}$-(4nitrofenil)benzenossulfonamida. É a primeira vez que estes derivados de sulfonamida são apresentados em associação com a pralidoxima como potenciais agentes terapêuticos para o tratamento da intoxicação por compostos organofosforados. Todas as associações avaliadas demonstraram capacidade de reativar a acetilcolinesterase humana recombinante previamente inibida por paraoxon nas doses avaliadas. As moléculas com maior potencial de reativação da atividade da acetilcolinesterase em associação com a pralidoxima foram $\mathrm{N}$-(2-clorofenil)benzenossulfonamida $\mathrm{e}$ $\mathrm{N}$-(4-fluorofenil)benzenossulfonamida.

Palavras chave: Pralidoxima; Paraoxon; Sulfonamida; Reativação; Acetilcolinesterase.

\begin{abstract}
(In vitro evaluation of the synergism between sulfonamide compounds and pralidoxime in the reactivation of recombinant human acetylcholinesterase inhibited by organophosphorus compound paraoxon). Eleven sulfonamide compounds were associated to pralidoxime and tested to determine their ability of reactivation of recombinant human acetylcholinesterase previously inhibited with $2 \mu \mathrm{M}$ Paraoxon solution. Each sulfonamide was used in $100 \mu \mathrm{M}$ solution at dimethylsulfoxide, such as $\mathrm{N}$-(2-chlorophenyl)benzenesulfonamide, $\mathrm{N}$-(4-fluorophenyl))benzenesulfonamide, $\mathrm{N}$-(4fluorophenyl)-4-nitrobenzenesulfonamide, 4-chloro- $\mathrm{N}$-(4-fluorophenyl)benzenesulfonamide $\mathrm{N}$-(3chlorophenyl)benzenesulfonamide, $\mathrm{N}$-(3-chloro-2-methylphenyl)benzenesulfonamide, 4-chloro- $\mathrm{N}$ phenylbenzenesulfonamide, $\mathrm{N}$-(2-chlorophenyl)-4-methylbenzenesulfonamide, $\mathrm{N}$-(3-chlorophenyl)4-methylbenzenesulfonamide, N-(2-hydroxyethyl)-4-methyl-N-phenylbenzenesulfonamide and $\mathrm{N}$ (4-nitrophenyl)benzenesulfonamide. It is the first time that these benzenesulfonamide derivatives are shown in association with pralidoxime as potential therapeutic agents for the treatment of intoxication by organophosphorus compounds. All evaluated compounds were able to reactivate acetylcholinesterase previously inhibited by paraoxon at the doses evaluated. The molecules with the
\end{abstract}




\section{ACIA \\ Biomedica Brasiliensia}

ISSN: 2236-0867

highest acetylcholinesterase reactivation potential in association with pralidoxime was $\mathrm{N}-(2$ chlorophenyl)benzenesulfonamide and N-(4-fluorphenyl)benzenesulfonamide.

Keywords: Pralidoxime; Paraoxon; Sulfonamide; Reactivation; Acetylcholinesterase.

\section{Introdução}

A pralidoxima é o fármaco indicado no tratamento de intoxicação por pesticidas e compostos organofosforados com atividade anticolinesterásica, atuando como antídoto para intoxicação por agentes organofosforados, que se ligam ao sítio esterático da acetilcolinesterase e que resulta inicialmente em uma inativação reversível da enzima (PETRONILHO, 2011).

Contraditoriamente, do ponto de vista do tratamento de intoxicação em humanos, no entanto, a análise de nove ensaios clínicos randomizados, envolvendo 638 (seiscentos e trinta e oito) pacientes no total, demonstrou que a utilização de oximas, em destaque, a pralidoxima, mesmo sob diferentes regimes terapêuticos, não se mostra mais eficiente em situações reais do que o placebo, concomitante à administração de atropina e suporte ventilatório durante a internação (BLAIN, 2011).

A pralidoxima, laboratorialmente, também retarda o processo de 'envelhecimento' da acetilcolinesterase fosforilada, que pode se tornar irreversivelmente inibida. O efeito mais importante que se espera deste fármaco é o tratamento da paralisia dos músculos respiratórios. Pelo motivo de a pralidoxima ser menos efetiva no alívio da depressão respiratória, a atropina é sempre requerida concomitantemente para bloquear o efeito da acetilcolina acumulada na membrana pós-sináptica. A pralidoxima tem sido relatada como tendo a atividade de aliviar os sinais e sintomas muscarínicos como salivação e broncoespasmo, mas esta ação é relativamente desimportante pois a atropina é mais adequada para este propósito terapêutico (POHANKA et al., 2009).

Foi relatado na literatura científica um derivado de benzenossulfonamida, 4-cloro-N-(4nitrofenil)benzenossulfonamida, que apresentou a efetiva capacidade de reativar a acetilcolinesterase inibida por compostos organofosforados em testes in vitro e in vivo em modelos animais, sendo testado contra o simulador de agente neurotóxico organofosforado diisopropilfluorofosfato. Tal composto foi adotado como modelo para a série de compostos sintetizados neste presente trabalho (BHATTACHARJEE et al., 2015; ABBASI et al., 2014).

$\mathrm{O}$ teste in vitro realizado neste trabalho consiste basicamente em avaliar o sinergismo da associação entre sulfonamidas e pralidoxima, indiretamente, por meio da verificação da capacidade da enzima acetilcolinesterase processar o substrato acetiltiocolina, utilizando para este intuito o método espectrométrico de Ellman, que quantifica a velocidade de hidrólise do substrato devido à interação do produto da hidrólise com o reagente ácido ditiobisnitrobenzóico, cujo ânion pode ser detectado espectrofotometricamente no comprimento de onda máximo $\left(\lambda_{\text {máx }}\right)$ de $412 \mathrm{~nm}$ (SILVA, 2012; PETRONILHO, 2011; RIBEIRO, 2009).

\section{Materiais e Métodos}

\subsection{Solventes, reagentes e instrumentos}

Foi utilizada enzima acetilcolinesterase humana recombinante do fabricante Sigma-Aldrich®, expressa em células HEK 293, com as seguintes especificações: 1000 UN/mg (1 UN hidrolisa $1 \mu$ mol de acetilcolina por min). Não foi utilizada solução estabilizante de albumina de soro bovina, pois foram preparadas várias alíquotas submetidas a congelamento em freezer $-80^{\circ} \mathrm{C}$, consumidos conforme a necessidade do experimento. Foi utilizado ácido ditiobisnitrobenzóico (DTNB), iodeto 


\section{ACTA \\ Biomedica Brasiliensia}

ISSN: 2236-0867

de acetiltiocolina, iodeto de pralidoxima e paraoxon do fabricante Sigma-Aldrich® e foi utilizado o leitor de microplacas por espectroscopia do fabricante Molecular Devices®, disponível no laboratório do Instituto de Biologia do Exército. Foram utilizados derivados de sulfonamida disponíveis no Laboratório de Síntese Orgânica do Instituto Militar de Engenharia na forma de solução a $100 \mu \mathrm{M}$ em dimetilsulfóxido: N-(2-clorofenil)benzenossulfonamida, N-(4-fluorofenil)benzenossulfonamida, N(4-fluorofenil)-4-nitrobenzenossulfonamida, 4-cloro-N-(4-fluorofenil)benzenossulfonamida, N-(3clorofenil)benzenossulfonamida, $\quad \mathrm{N}$-(3-cloro-2-metilfenil)benzenossulfonamida, 4-cloro-Nfenilbenzenossulfonamida, $\mathrm{N}$-(2-clorofenil)-4-metilbenzenossulfonamida, $\mathrm{N}$-(3-clorofenil)-4metilbenzenossulfonamida, $\quad \mathrm{N}$-(2-hidroxietil)-4-metil-N-fenilbenzenossulfonamida e $/ \mathrm{N}-(4-$ nitrofenil)benzenossulfonamida.

\subsection{Inibição da acetilcolinesterase humana recombinante com paraoxon}

Em cada poço de uma microplaca de 96 poços foram adicionados $20 \mu \mathrm{L}$ de solução de AChE (0,5 UN/mL), $5 \mu \mathrm{L}$ de solução de DTNB $(0,01 \mathrm{M})$. $100 \mu \mathrm{L}$ de tampão fosfato $0,1 \mathrm{M}$ pH 7,4, $55 \mu \mathrm{L}$ de uma solução de organofosforado, em concentração variando de $10 \mu \mathrm{M}$ a $0,1 \mu \mathrm{M}$ de Paraoxon, sendo por último realizada a adição de $20 \mu \mathrm{L}$ de solução de iodeto de acetiltiocolina $0,005 \mathrm{M}$.

2.3 Ensaio de atividade in vitro da reativação da acetilcolinesterase humana recombinante inibida com paraoxon com derivados de sulfonamida em associação com a pralidoxima por espectroscopia de ultravioleta-visível

Conforme metodologia utilizada por Petronilho (2011) e Silva (2012), o teste de reativação da acetilcolinesterase foi realizado através da adição de $20 \mu \mathrm{L}$ de solução de AChE $(0,5 \mathrm{UN} / \mathrm{mL}), 5 \mu \mathrm{L}$ de solução de DTNB (0,01 M), tampão fosfato $0,1 \mathrm{M} \mathrm{pH} \mathrm{7,4} \mathrm{(até} \mathrm{perfazer} 200 \mu \mathrm{L}$ no volume final), $100 \mu \mathrm{L}$ de solução de paraoxon $(2 \mu \mathrm{M})$, e após $60 \mathrm{~min}$, é feita a adição de $10 \mu \mathrm{L}$ da solução de derivado de benzenossulfonamida a $100 \mu \mathrm{M}$ e de $10 \mu \mathrm{L}$ da solução de pralidoxima a $100 \mu \mathrm{M}$ e por último foi adicionado $20 \mu \mathrm{L}$ de solução de iodeto de acetiltiocolina $0,005 \mathrm{M}$, respectivamente, em cada um dos poços de uma microplaca de 96 poços, onde imediatamente após a adição do substrato, a microplaca foi levada ao espectrofotômetro e a leitura foi iniciada com amostragem a cada 5 min em um tempo total de $15 \mathrm{~min}$ ), fazendo-se uso do leitor de microplacas por espectroscopia. Foram realizados quatro repetições para cada poço (quadruplicata) e baseando-se na velocidade máxima obtida a partir da regressão linear de todos os pontos obtidos pela leitura das quadruplicatas no comprimento de onda disponível no equipamento utilizado $(\lambda=406 \mathrm{~nm})$, foi calculado o potencial de interferência das diversas substâncias na atividade da acetilcolinesterase.

\section{Resultados}

Após a realização do experimento de inibição da acetilcolinesterase recombinante humana com paraoxon, foi verificada que a solução de paraoxon a $2 \mu \mathrm{M}$ era suficiente para atingir inibição total da atividade da enzima nas condições experimentais adotadas neste estudo e que a solução de pralidoxima a $100 \mu \mathrm{M}$ era suficiente para reativar a atividade da acetilcolinesterase após a inibição. A partir dos resultados da avaliação in vitro dos derivados de benzenossulfonamida como reativadores da acetilcolinesterase inibida por paraoxon, foi possível verificar a atividade de moléculas estruturalmente semelhantes à molécula citada por Bhattacharjee et al. (2015). Os resultados se encontram descritos na Tabela 1. 
Tabela 1: Teste de reativação da acetilcolinesterase inibida por paraoxon com os derivados de benzenossulfonamida em associação com a pralidoxima (Continua).

\begin{tabular}{cc}
\hline \multirow{2}{*}{ MOLÉCULAS TESTADAS } & TAXA DE REATIVAÇÃO COM \\
SULFONAMIDAS A 100 $\mu$ EM EM \\
ASSOCIAÇÃO COM A PRALIDOXIMA A \\
$100 \mu M$
\end{tabular}
$100 \mu \mathrm{M}$

\section{PARAOXON $2 \mu \mathrm{M}$}

PRALIDOXIMA $100 \mu \mathrm{M}$

$\mathrm{N}$-(2-clorofenil)benzenossulfonamida<smiles>O=S(=O)(Nc1ccccc1Cl)c1ccccc1</smiles>

$\mathrm{N}$-(4-fluorofenil)benzenossulfonamida<smiles>O=S(=O)(Nc1ccc(F)cc1)c1ccccc1</smiles>

$\mathrm{N}-(4-$ fluorofenil)-4nitrobenzenossulfonamida<smiles>O=[N+]([O-])c1ccc(S(=O)(=O)Nc2ccc(F)cc2)cc1</smiles>

4-cloro-N-(4-

fluorofenil)benzenossulfonamida<smiles>O=S(=O)(Nc1ccc(F)cc1)c1ccc(Cl)cc1</smiles>

$\mathrm{N}$-(3-clorofenil)benzenossulfonamida<smiles>O=S(=O)(Nc1cccc(Cl)c1)c1ccccc1</smiles>

$\mathrm{N}$-(3-cloro-2-

metilfenil)benzenossulfonamida<smiles>Cc1c(Cl)cccc1NS(=O)(=O)c1ccccc1</smiles>

4-cloro-N-fenilbenzenossulfonamida<smiles>O=S(=O)(Nc1ccccc1)c1ccc(Cl)cc1</smiles>

$0 \%$

$100 \%$

$127 \%$

$122 \%$

$75 \%$

$87,5 \%$

$81,25 \%$

$72,92 \%$

$93,75 \%$ 
Tabela 1: Teste de reativação da acetilcolinesterase inibida por paraoxon com os derivados de benzenossulfonamida em associação com a pralidoxima (Conclusão).

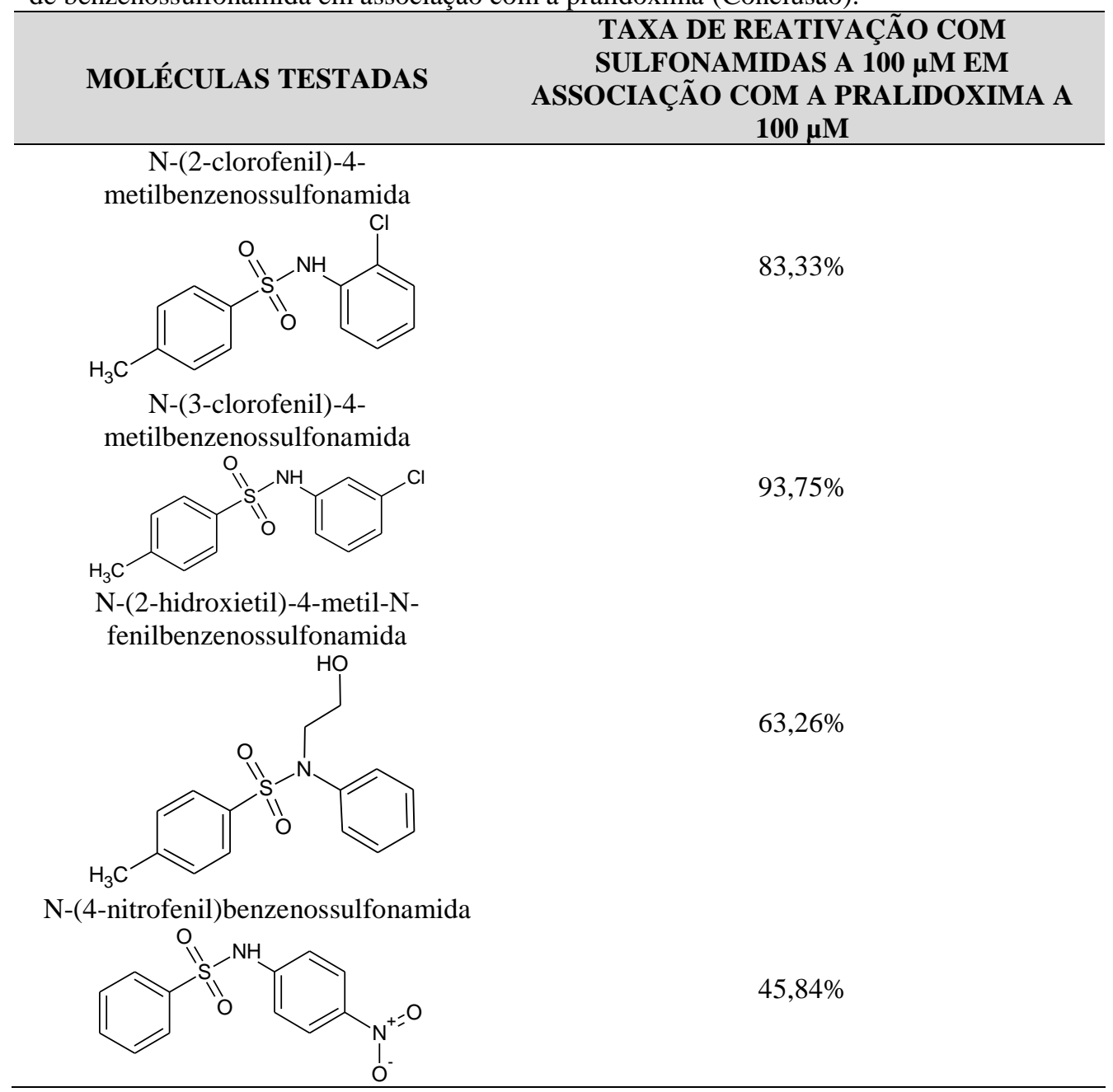

\section{Discussão}

Todos os derivados de sulfonamida avaliados em associação com a pralidoxima demonstraram capacidade de reativar a acetilcolinesterase previamente inibida por paraoxon nas doses avaliadas, embora em nível muito inferior à pralidoxima, em alguns casos, o que permite inferir a necessidade de uma dose em concentração maior, para atingir efeitos farmacológicos semelhantes.

Os derivados de sulfonamida testados em associação à pralidoxima têm aumento significativo na taxa de reativação normal quando apenas pralidoxima é utilizada, com destaque para os compostos $\mathrm{N}$-(2-clorofenil)benzenossulfonamida e $\mathrm{N}$-(4-fluorofenil)benzenossulfonamida, que provocaram um aumento de $27 \%, 22 \%$ e $27 \%$ na taxa de reativação com sulfonamidas a $100 \mu \mathrm{M}$ em associação com a pralidoxima a $100 \mu \mathrm{M}$, respectivamente, conforme já foi previamente demonstrado na Tabela 1.

\section{Conclusão}

Considerando a acetilcolinesterase como importante alvo terapêutico em função do seu envolvimento na manutenção do funcionamento do sistema nervoso central e periférico, 
fundamentalmente, nas terminações nervosas colinérgicas, sugere-se continuar a buscar um mais completo entendimento da ação das sulfonamidas, que apenas recentemente têm recebido atenção no que se refere às suas possibilidades terapêuticas no tratamento de intoxicação por compostos organofosforados.

É a primeira vez que estes derivados de sulfonamida, com estrutura baseada no recente trabalho de Bhattacharjee et al. (2015) são testados em associação com a pralidoxima para verificar seu potencial de sinergismo in vitro como potenciais agentes terapêuticos para o tratamento da intoxicação por compostos organofosforados.

\section{Referências}

ABBASI, M., AHMAD, S., REHMAN, A., RASOOL, S., KHAN, K., ASHRAF, M., NASAR, R. \& ISMAIL, T. 2014. Sulfonamide Derivatives Of 2-Amino-1-Phenylethane As Suitable Cholinesterase Inhibitors. Tropical Journal of Pharmaceutical Research , v.13, 739.

BHATTACHARJEE, AK. et al. 2015. Discovery of non-oxime reactivators using an in silicopharmacophore model of reactivators for DFP-inhibited acetylcholinesterase. European Journal Of Medicinal Chemistry, v. 1, n. 90: 209-220. 2015.

BLAIN, P. G. 2011. Organophosphorus poisoning (acute). BMJ Clinical Evidence, v.1: 2102-2119. 2011.

PETRONILHO, E.C. 2011. Síntese e Avaliação de Hidrazonas Como Inibidoras e Reativadoras da Acetilcolinesterase. 110f. Dissertação de mestrado (Química) - Instituto Militar de Engenharia (IME), Rio de Janeiro, 2011.

POHANKA, M.; JUN, D. \& KUCA, K. 2009. In vitro reactivation of trichlorfon-inhibited butyrylcholinesterase using HI-6, obidoxime, pralidoxime and K048. J Enzyme Inhib Med Chem., Hradec Kralove, v. 3: 680-683, jun. 2009.

RIBEIRO, T.S. 2009. Síntese e Avaliação de Novas Oximas como Antídotos de Intoxicação por Organofosforados. 108f. Tese de Doutorado (Química) - Instituto Militar de Engenharia (IME), Rio de Janeiro, 2009.

SILVA, G. R. 2012. Síntese e avaliação de novas séries de oximas e hidrazonas como potenciais reativadores da enzima acetilcolinesterase inibida por organofosforados neurotóxicos. $217 \mathrm{f}$. Tese de Doutorado (Química) - Instituto Militar de Engenharia, Rio de Janeiro, 2012. 